\title{
Cueva Alí Mustafá: un nuevo sitio arqueológico con ocupación temprana en las Sierras Orientales de Tandilia (Argentina)
}

\section{Diana L. Mazzanti", Gustvo F. Bonnat" ${ }^{* *}$, Juan P. Donadei Corada" y Gustavo A. Martínez ***}

\section{Resumen}

Se exponen los resultados preliminares de los trabajos arqueológicos realizados en la Cueva Alí Mustafá. Este sitio se suma a otros reparos rocosos usados por grupos de cazadores-recolectores bajo la modalidad de ocupaciones de corta duración ocurridas durante la transición Pleistoceno-Holoceno. La información que incorpora este nuevo sitio temprano apoya la propuesta anterior sobre el valor arqueológico que tuvieron los reparos rocosos que poseen ocupaciones efímeras. En algunos casos presentan ocupaciones redundantes y se localizan en relación estratégica con el paisaje serrano. Por su localización con alta visualización del valle de San Pedro, este sitio fue utilizado como paradero durante las travesías por los valles serranos.

\section{Ali Mustafá Cave: a new archaeological site with an early occupation in the Eastern Tandilia Mountain Range (Argentina)}

Recibido: 22 de marzo de 2018

Aceptado: 17 de septiembre de 2018

\section{Palabras clave}

Sierras de Tandilia oriental Cazadores-recolectores tempranos

Transición Pleistoceno-Holoceno

\begin{abstract}
Here we present the preliminary results of the archaeological research in Ali Mustafá Cave. This site is one of several rockshelters used by hunter-gatherer groups for shortterm occupations during the Pleistocene-Holocene transition. The data from this new early site supports previous research regarding the archaeological value of rock shelters with ephemeral occupations. In some cases, these shelters show evidence of recurrent occupations, and were located in a strategic position in relation with the surrounding

\footnotetext{
* Laboratorio de Arqueología Regional Bonaerense (LARBO), Facultad de Humanidades, Universidad Nacional de Mar del Plata (UNMdP). Av. Juan B. Justo 2550 (CP B7608FBY), Mar del Plata, Buenos Aires, Argentina. E-mail: arqueolab@gmail.com

** Laboratorio de Arqueología Regional Bonaerense (LARBO), Facultad de Humanidades, Universidad Nacional de Mar del Plata (UNMdP) - CONICET. Av. Juan B. Justo 2550 (CP B7608FBY), Mar del Plata, Buenos Aires, Argentina. E-mail: fbonnat@hotmail.com; paolodonadeicorada@gmail.com

*** Instituto de Geografía de Costas y del Cuaternario (IGCyC), Universidad Nacional de Mar del Plata (UNMdP) - Comisión de Investigaciones Científicas de la Provincia de Buenos Aires (CIC), Dean Funes 3350 (CP B7602), Mar del Plata, Buenos Aires, Argentina. E-mail: gustavo.gmarti@gmail.com
}

\section{Keywords}

Eastern Tandilia range Early hunter-gatherers Pleistocene-Holocene transition 
mountain landscape. Due to its location with high visual dominance over the San Pedro Valley, this site was used as a stopover during movement through the intermontane valleys.

\section{Introducción}

La puesta en marcha de una arqueología de carácter regional en las sierras del borde oriental de Tandilia (Figura 1) permitió reconstruir la secuencia arqueológica local. Un ejemplo de ello, y de la importancia de los sitios arqueológicos en reparos rocosos, proviene de la localidad arqueológica Amalia, donde se conservaron las evidencias de varias ocupaciones indígenas sucesivas en la cueva del Sitio 2, que abarcan desde ca.10700 años AP hasta finales del siglo XVIII (Mazzanti, 2007).

Esto plantea la importancia que tuvo la microrregión de Tandilia oriental en la cual se analizan diversas problemáticas arqueológicas desde enfoques interdisciplinarios, que abarcan estudios zooarqueológicos, geoarqueológicos, paleobotánicos, paleoambientales y arqueométricos desde hace varias décadas (Bonnat, 2016; Colobig, 2011; Luna, Aranda y Quintana, 2017; Martínez 2007; Martínez y Mazzanti, 2017; Martínez et al., 2013; Mazzanti, 2007; Mazzanti et al., 2013; Mazzanti y Porto López, 2007; Mazzanti y Quintana, 2001; Quintana, 2016; Zucol, Brea, Osterrieth y Martínez, 2002, entre otros).

Esta área de investigación reúne evidencias significativas sobre el poblamiento temprano de la región pampeana a partir del estudio de los contextos de once reparos rocosos, en los cuales se registraron evidencias de actividades humanas características de los campamentos residenciales y otros de actividades específicas de corta duración. Todos estos sitios presentan cronologías asignadas a la transición Pleistoceno-Holoceno temprano (Bonnat, 2016; Mazzanti y Bonnat 2013). En este marco de investigación local, el objetivo de esta contribución es dar a conocer los primeros resultados obtenidos en el sitio Cueva Ali Mustafá, enfatizando el análisis del registro arqueológico de la ocupación humana más temprana.

\section{Cueva Alí Mustafá. Antecedentes sobre su descubrimiento}

Este sitio arqueológico fue citado en trabajos anteriores como Cueva Mustafá (Mazzanti, 1995-96) tomando el apellido de un inmigrante apodado el "Turco" Alí Mustafá. Los autores de este trabajo decidieron utilizar su nombre completo con el fin de diferenciar este sitio de otra cueva que fue denominada Cueva Mustafá que integra el Sitio 2 de la Localidad Arqueológica Amalia (Bonnat, Mazzanti y Martínez, 2015).

Alí Mustafá vivió en el área geográfica de Balcarce durante los primeros años de la década de 1920, desde entonces es considerado un representante del fenómeno definido por Chumbita (2000) como bandolerismo social. Mustafá se convirtió en un personaje de la historia local por los hurtos que cometió y por refugiarse en cuevas de las sierras cercanas a esa ciudad. Tuvo dos eventos de fuga de la policía y finalmente fue detenido en proximidades de lo que hoy es el sitio arqueológico Cueva Tixi ${ }^{1}$. Su popularización fue tal que otras cuevas en las sierras del Partido de Balcarce fueron apodadas con su nombre (e.g. Amalia Sitio 2). Los pobladores rurales, aún sostienen la creencia que las cuevas fueron los lugares donde pudo enterrar las supuestas joyas robadas. La vida de Mustafá fue el argumento de leyendas populares que dinamizan el folklore local, al punto de haber nutrido la trama de una novela de ficción (Dágata, 1987). Este texto literario es utilizado en el sistema escolar de Balcarce y fuente de mitos que pudieron 


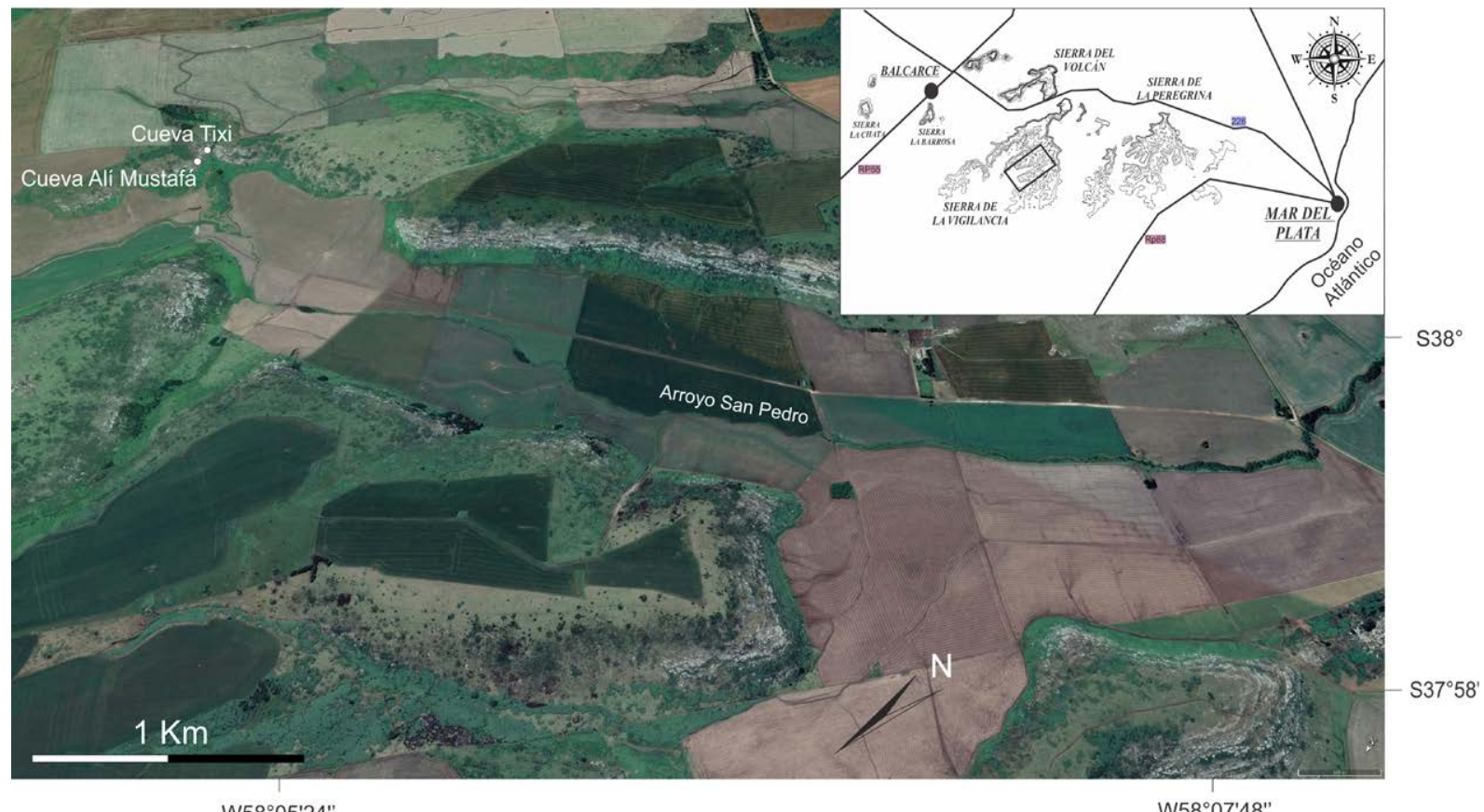

Figura 1. Localización de la Cueva Ali Mustafá en la cercanía de la naciente del arroyo San Pedro.

estimular la acción de aficionados de esa localidad en la búsqueda de los imaginarios tesoros enterrados por Mustafá.

El descubrimiento de este sitio ocurrió en 1982 cuando se realizaban los primeros registros y sondeos en Cueva Tixi, que dista $100 \mathrm{~m}$ aguas arriba sobre la misma pendiente. Su hallazgo fue casual porque dos bloques grandes desprendidos del techo obstruyeron su frente determinando una visibilización (Criado Boado, 1999) prácticamente nula (Figuras 2 y 3 ).

Los primeros trabajos arqueológicos se centraron en la realización de un sondeo de 40 x $40 \mathrm{~cm}$ y en el levantamiento de un gráfico de planta. En 1992 el geólogo M. Zárate y el Sr. C. Quintana reconocieron aspectos estratigráficos y faunísticos de los dos sitios arqueológicos mencionados. Se efectuaron dos nuevos sondeos reconociéndose la capa superior laminar, presencia de carbón y algunos artefactos líticos en una unidad sedimentaria inferior.

A pesar de estos resultados preliminares, el estudio sistemático de esta cueva fue postergado varias décadas debido a la importancia y magnitud de otros registros arqueológicos que requerían de investigaciones inmediatas, como fueron Cueva Tixi y otros reparos rocosos que se iban descubriendo en esos años (Abrigo Los Pinos, Cueva Burucuyá, Cueva El Abra, Cueva La Brava, Localidad arqueológica Amalia, entre otros). En la actualidad y en el marco de dos proyectos de investigación subsidiados (ANPCYT-PICT 2013 y UNMDP15/F533) se retomó el estudio de los sitios con ocupaciones efímeras registrados en las décadas anteriores (Cueva Mustafá, Alero La Cautiva, Rincón Grande y Molina I, etc.).

\section{Consideraciones metodológicas}

Los trabajos en Cueva Alí Mustafá se realizaron en dos campañas (2017 y 2018) mediante excavaciones en área extrayendo las unidades sedimentarias naturales por medio de decapados y registros en plantas y secciones estratigráficas (Mazzanti, 1993). Por la 


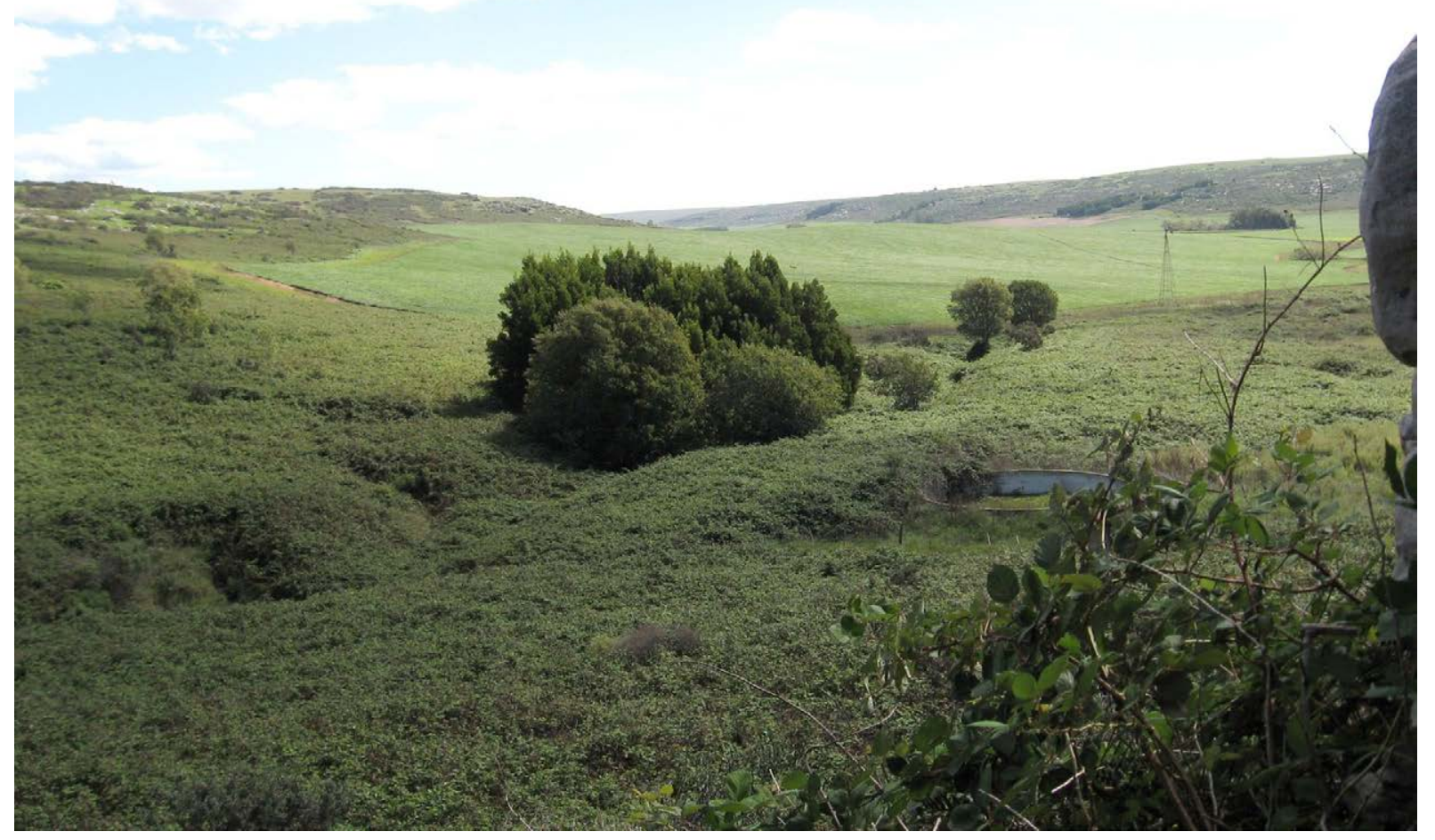

Figura 2. Valle del arroyo San Pedro visto desde el vestíbulo de la Cueva Ali Mustafá.
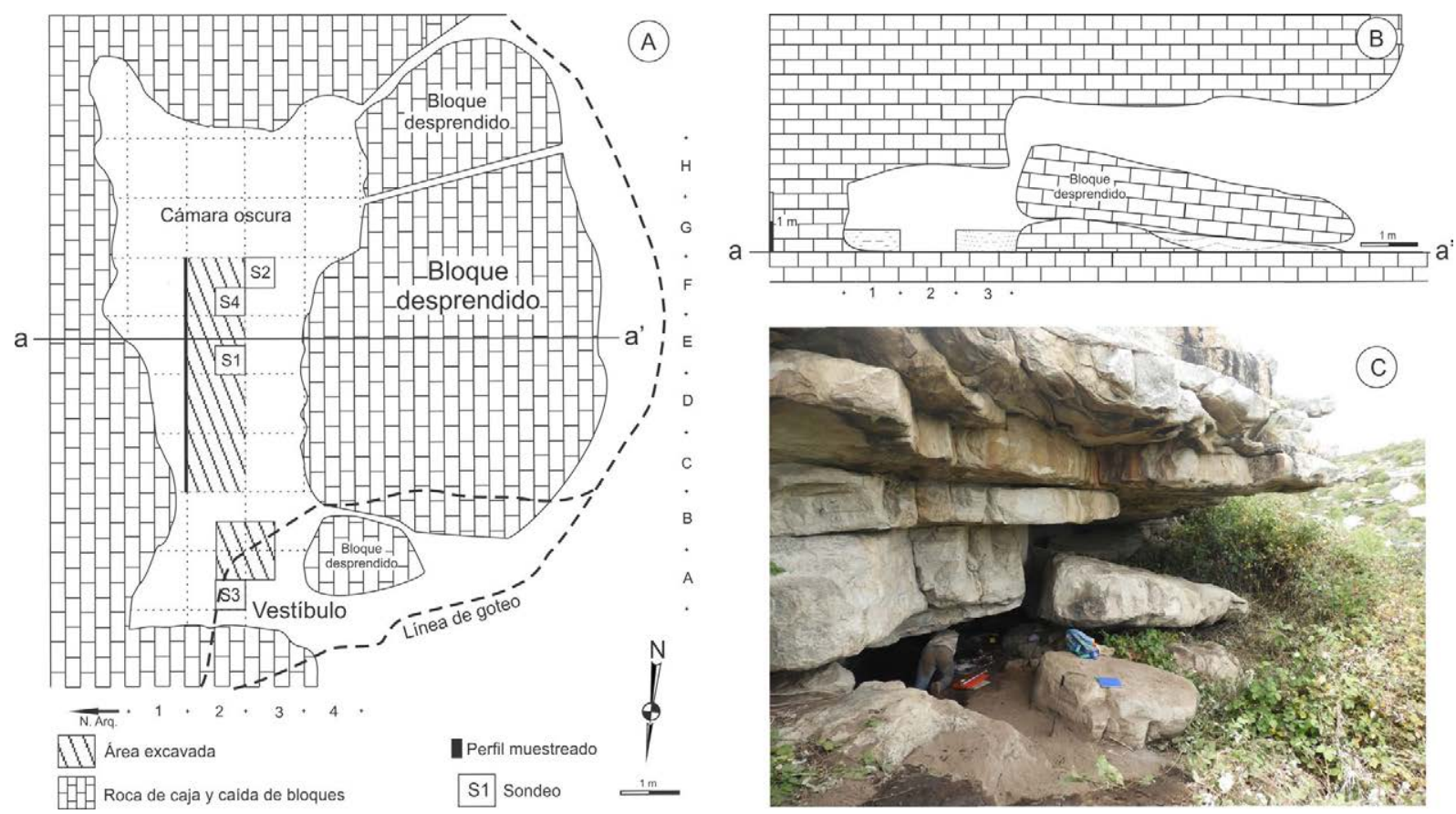

Figura 3. A) Plano de planta del sitio Cueva Ali Mustafá. B) Corte transversal de la cueva. C) Vista del sitio con su entrada lateral.

buena conservación de los depósitos estratificados se extrajo un testigo de la secuencia completa con el objetivo de realizar estudios geoarqueológicos y paleoambientales mediante análisis de micromorfología. 
Con respecto a la metodología utilizada en el análisis preliminar del conjunto lítico se consideraron las propuestas de Aschero $(1975,1983)$ y Bellelli, Guraieb y García (1985-1987). Las variables seleccionadas corresponden a la identificación de los tipos de rocas y a la clasificación de los artefactos en categorías (e. g. núcleos, instrumentos, desechos y microdesechos de talla). Por otra parte, se relevaron presencia/ausencia de corteza y variables métricas.

Las cronologías se obtuvieron por medio de ${ }^{14} \mathrm{C}$ (AMS) procesadas en la Universidad de Arizona (USA), sobre muestras de carbón obtenidas en los rellenos de las cubetas y de trozos pequeños de carbón dispersos recolectados en las unidades superiores.

\section{Cuenca de ocupación del valle de San Pedro}

El valle formado por el arroyo San Pedro tiene una longitud de ocho kilómetros. Su naciente se ubica en el interior del macizo de la sierra La Vigilancia y está formado por pendientes suaves con presencia de cuevas y aleros (Figuras 1 y 2 ). Este paisaje se complementa con las planicies extensas que caracterizan la cima serrana mesetiforme. Dos cursos fluviales se originan en la cumbre y se unen dando origen al arroyo San Pedro, en el cual desaguan cursos más pequeños. La Cueva Alí Mustafá se localiza en un extremo de una pequeña quebrada tributaria.

Esta cueva posee relación de vecindad con otros reparos localizados recientemente en el mismo valle por dos de los autores (G. F. B. y J. P. D.). Se trata de tres aleros emplazados en la vertiente norte del valle con materiales líticos en capa y en superficie, que se integran al conjunto de otros sitios descubiertos en el valle vecino (Arroyo La Vigilancia) con ocupaciones datadas en la transición Pleistoceno-Holoceno (Bonnat, 2016). Otros sitios, en ese valle, se registraron en reparos muy pequeños con ocupaciones de muy corta permanencia (Alero Lobuno y Alero El Limón). Estos sitios con un bajo número de materiales y pocas posibilidades de obtener dataciones, brindaron cobijo durante los derroteros por los valles. Estos hábitats pequeños fueron eficientes para ciertas actividades específicas o bien por las ventajas que ofrecían sus localizaciones en el paisaje.

\section{Características del sitio}

La Cueva Alí Mustafá se localiza a los 3758'23,7” S - 5804’10” O, a una altura de $171 \mathrm{~m}$ s.n.m., en el extremo noreste de una pequeña quebrada con curso de agua permanente que desemboca en el arroyo San Pedro. Este pequeño tributario se origina a $700 \mathrm{~m}$ sobre la cima serrana (Figura 1). Su ventajosa ubicación en el paisaje serrano le otorgó a esta cueva condiciones estratégicas para su ocupación, porque controla visualmente gran parte de la cuenca del valle de San Pedro (Figura 2) y por otra parte posee baja visibilización.

La caída de dos grandes bloques del techo del alero exterior obturó por completo la entrada produciendo una cavidad longitudinal con techo muy bajo y con muy poco ingreso de luz natural, por ello su entrada es lateral y posee dos techos con distintas extensiones (cueva y alero) (Figura $3 \mathrm{~A}$ y C). Estas características de origen geológico debieron limitar las actividades antrópicas que pudieran realizarse en su interior.

La cámara bajo el techo más amplio integra a dos sectores: uno externo o vestíbulo abierto con alero y buena insolación y otro interno que corresponde a la cueva propiamente dicha y con un piso amplio colmatado por sedimentos. Actualmente este techo es muy bajo reduciendo las posibilidades de la circulación humana en posición erguida (Figura 3B y C), circunstancia que pudo mejorar durante la primera ocupación humana. 
2. El análisis de las muestras de diatomeas de las Unidades 1 y 2 está a cargo de la Dra. G. Hassan (CONICET-UNMDP).
El ingreso de agua pluvial ocurre por dos vías, una desde la boca lateral y otra por una vertiente del muro posterior, las que humedecen algunos sectores de la matriz sedimentaria produciendo además la concentración de sedimentos con diatomeas.

\section{Rasgos geoarqueológicos}

La matriz sedimentaria del sitio corresponde a una secuencia condensada (sensu Martínez y Mazzanti, 2017) con un espesor variable entre $0,40 \mathrm{~m}$ en el fondo de la cueva y $0,50 \mathrm{~m}$ en su exterior. Dicha secuencia presenta cambios faciales laterales (Figura $4 \mathrm{~A} \mathrm{y} \mathrm{C).} \mathrm{En} \mathrm{la} \mathrm{cámara} \mathrm{interior} \mathrm{se} \mathrm{identificaron} \mathrm{cinco} \mathrm{unidades} \mathrm{estratigráficas}$ sobre la base de características sedimentarias (textura, color, forma, tipo de límites, estructuras sedimentarias y grado de compactación) (Figura 4B).

La unidad inferior (U5) corresponde a depósitos de edad Pleistoceno tardío, observados en numerosos sitios de Tandilia oriental (Martínez y Mazzanti, 2017). Estos se destacan por su granulometría más gruesa (areno-limosa) y color castaño claro. El contacto con la U4 es discordante y de forma ondulada. La discordancia se ubica inmediatamente por debajo de los niveles de ocupación temprana (ca.9400-10500 años AP), coincidente con el mejoramiento climático postglacial a partir del cual ingresaron las sociedades humanas a esta región serrana (Martínez et al., 2013). En esta unidad se registraron numerosos fogones en cubeta (Figura $4 \mathrm{E} \mathrm{y} \mathrm{F).} \mathrm{Las} \mathrm{unidades} \mathrm{superiores} \mathrm{holocenas} \mathrm{(U4,}$ U3, U2 y U1) presentan granulometría más fina (limo arcillo-arenosa) y tonalidades más oscuras, probablemente debido al incremento de materia orgánica. Se destaca la unidad 2 compuesta por lentes de limos diatomáceos y de diatomitas ${ }^{2}$, que se atenúan considerablemente hacia la boca de la cueva. Niveles similares fueron identificados en los tramos superiores de las secuencias correspondientes al Holoceno tardío (ca. 3.000 años AP) de numerosos sitios de la región (Cueva Burucuyá, Sitio 1 de la Localidad arqueológica Lobería I, Cueva La Brava y Cueva Tixi). Estos depósitos representan condiciones de mayor humedad en el recinto, que dependería de un aumento del régimen pluviométrico en la región para el Holoceno tardío (Martínez, 2007; Martínez et al., 2013).

Por otro lado, la cuadrícula dispuesta en el vestíbulo presenta características estratigráficas homogéneas en color y textura. Durante el trabajo de campo no se pudieron diferenciar claramente las unidades que componen ese sector de la matriz (Figura 4C). Se reconocen de modo preliminar tres unidades sedimentarias por la presencia de clastos y bloques.

\section{Registro arqueológico y cronología}

Se identificaron ocupaciones humanas efímeras asignadas a dos momentos del Holoceno y otras de características redundantes durante a la transición Pleistoceno tardío-Holoceno temprano (Tabla 1).

En este trabajo se mencionan sintéticamente algunas características de estos contextos, con especial atención a la ocupación temprana.

\section{Ocupación del siglo XX}

A lo largo de ese siglo la cueva fue usada como refugio por ocupantes ocasionales. En su entrada se halló un pequeño alineamiento de pircas creado como cerramiento de una grieta entre bloques. En el área cercana a la boca se halló un contexto moderno integrado al depósito sedimentario superficial, con un fogón asociado a restos industriales (botón, trozos de tela y vidrio) y fragmentos óseos de Myocastor coypus, Axis axis y fragmentos de diáfisis. En la superficie del piso actual se recolectaron fragmentos de loza con decoración gris. En el techo y paredes del alero exterior se conservan trazos muy deteriorados de inscripciones modernas. 

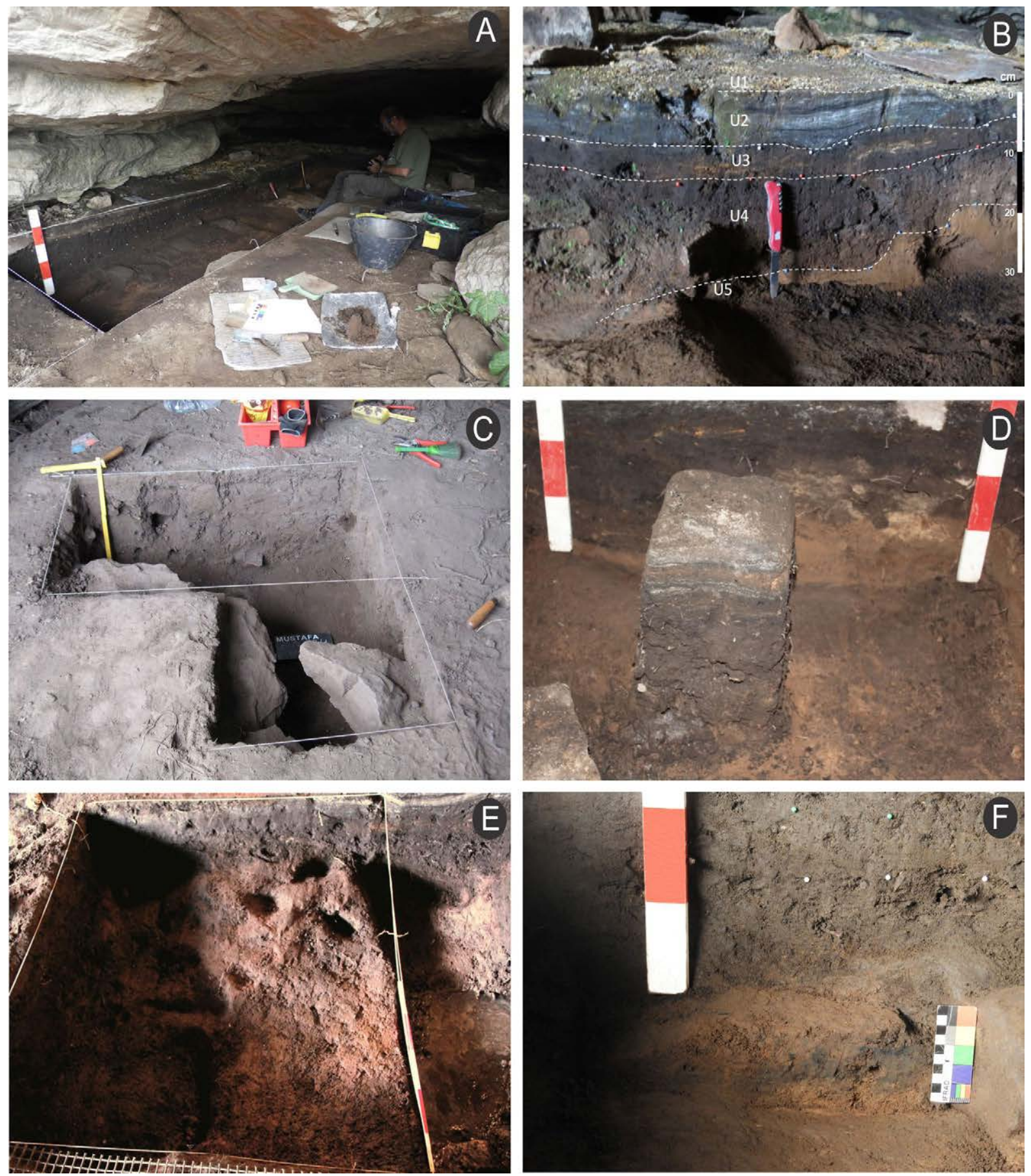

Figura 4. A) Imagen general del área excavada en el interior de la cueva. B) Unidades sedimentarias en el perfil de las cuadrículas E2-F2. C) Perfil del sector del vestíbulo, cuadrículas $A 2$ y B2. D) Testigo estratigráfico extraído de la cuadrícula F2. E) Detalle de las cubetas de los fogones, cuadrícula E2. F) Base del perfil que muestra el corte vertical de un fogón, cuadrícula C2.

\section{Ocupaciones Prehispánicas}

2.1. En la unidad sedimentaria superior caracterizada por sedimentos laminares, se hallaron pocas lascas de ortocuarcitas y espículas de carbón disperso. Una datación señala que este evento de una breve ocupación se produjo en el Holoceno tardío-final (Tabla 1). 
3. Por la Dra. Mariana Brea (Laboratorio de Paleobotánica del Centro de Investigaciones Científicas y Transferencia de Tecnología a la Producción, Entre Ríos).

\begin{tabular}{|c|c|c|c|}
\hline № de laboratorio & Años radiocarbónicos & Unidad estratigráfica & Tipo de muestras \\
\hline AA108365 & $401 \pm 19$ & $U_{2}$ & Trozo de carbón \\
AA110081 & $9060 \pm 31$ & $U_{4}$ & Carbón de fogón \\
AA110082 & $9032 \pm 30$ & $U_{4}$ & Carbón de fogón \\
AA108366 & $10125 \pm 31$ & $U_{4}$ & Carbón de fogón \\
AA110084 & $10450 \pm 38$ & $U_{4}$ & Carbón de fogón \\
AA110083 & $7670 \pm 30$ & $(?)$ & Trozo de carbón \\
\hline
\end{tabular}

Tabla 1. Dataciones por ${ }^{14} \mathrm{C}$ (AMS).

2.2. En el sector del vestíbulo (Figura 4C y $5 \mathrm{~A}$ ) se conservaron materiales líticos producidos por acciones de talla con características distintas al conjunto recuperado del interior de la cueva. Este grupo de materiales líticos se ubican cronológicamente a inicios del Holoceno medio (Tabla 1).

2.3. Dentro de la cueva, en la Unidad 4 (Figura 4B) se hallaron las evidencias de la ocupación humana más significativa en términos arqueológicos. Se identificaron nueve fogones en cubeta con abundantes fragmentos de carbón concentrados en su relleno y también se recolectó el carbón disperso. Se trata de indicadores de la ejecución de actividades de combustión intensas, reiteradas y de mantenimiento de las mismas (Figura 4E, 4F y 5). Los fragmentos mejor preservados están siendo analizados desde el punto de vista antracológico ${ }^{3}$ y los resultados complementarán la información sobre recursos vegetales existentes en estas cuencas serranas durante el postglaciar (Brea, Mazzanti y Martínez, 2014). Los sedimentos del área de combustión presentaron una coloración más clara y mayor consolidación y numerosos carbones dispersos. El área elegida para producir estas numerosas estructuras de combustión es coincidente con el sector de la cueva donde el techo es más bajo, lo que pudo generar situaciones de humaredas internas.

\section{Conjunto lítico}

El conjunto lítico de todo el sitio está compuesto por 515 artefactos (Tabla 2), los cuales pudieron ser asignados a los tres periodos cronológicos: cinco artefactos en los niveles superiores de las cuadrículas D2 y F2 (Holoceno tardío), 105 elementos recuperados en las cuadrículas del ingreso a la cueva (A2, B2 y B3) asignados al Holoceno medio y 405 artefactos procedentes de las cuadrículas C2, D2, E2 y F2 en el contexto datado en la transición Pleistoceno/Holoceno.

A los fines de este trabajo, sólo presentaremos un análisis preliminar del componente temprano (Tabla 3). Este conjunto de 405 artefactos fue agrupado en cuatro clases artefactuales: núcleos $(\mathrm{n}=1)$, lascas $(\mathrm{n}=306)$, microlascas $(\mathrm{n}=97)$ e instrumentos ( $\mathrm{n}$ $=1$ ). Un aspecto relevante que presenta el conjunto lítico es la diversidad de materias primas que comprende ocho variedades de rocas (Tabla 3). Las ortocuarcitas son las rocas más abundantes con el $92,11 \%$, integradas tanto por las del Grupo Sierra Bayas (OGSB) y de la Formación Balcarce (OFB). Dentro de estas dos variedades de rocas, se aprecia que la de mayor frecuencia es la variedad de grano fino (OGSB) no local con el $56,05 \%$. En esta roca se destaca la presencia de un instrumento, lascas pequeñas sin remanentes de corteza y microdesechos de talla.

Por otro lado, las OFB son el segundo recurso con mayor frecuencia identificado en el sitio (36,06\%). Sobre esta roca de grano medio-grueso y de disponibilidad local se observa una mayor presencia de lascas en relación con las microlascas (Tabla 3). 
(A)
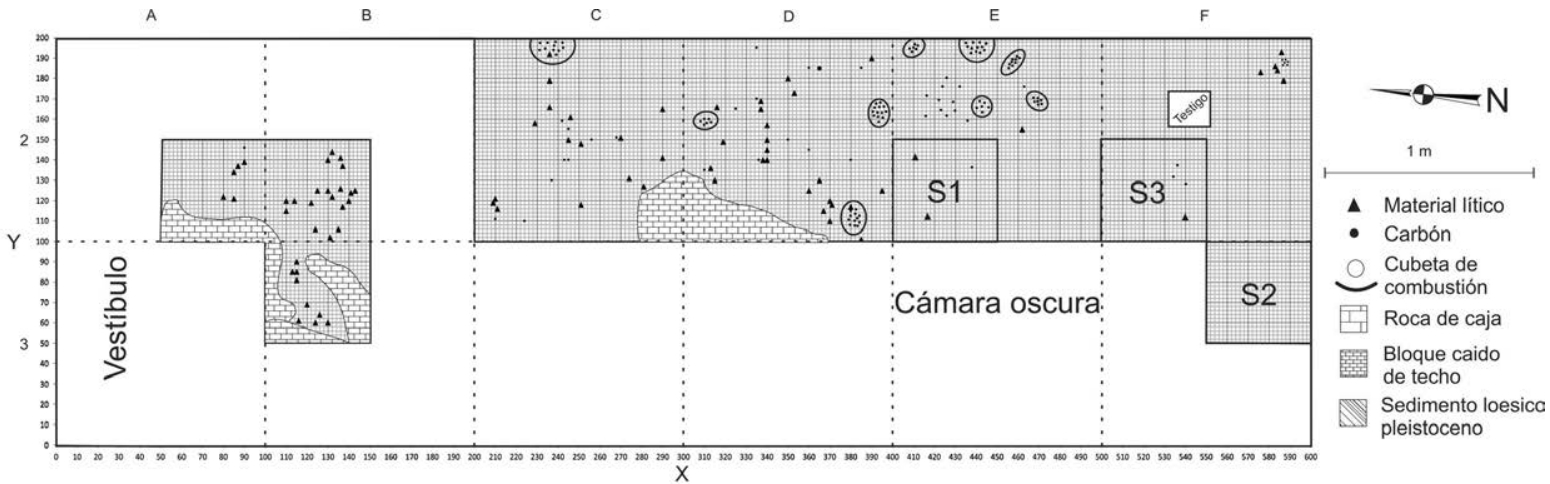

(B)

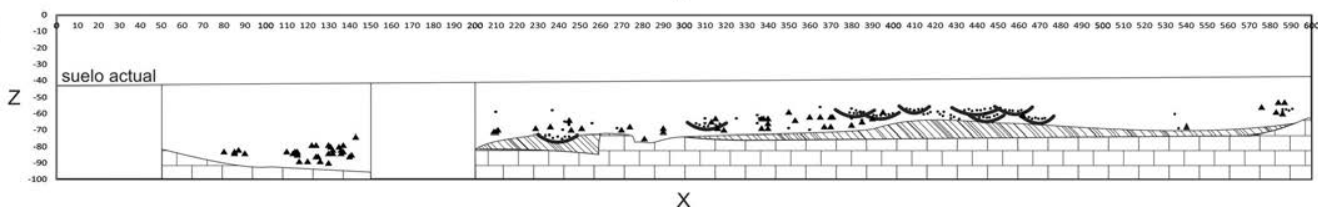

Figura 5. Cueva Ali Mustafá. A) Planta del área de excavación. B) Perfil de excavación con las áreas de actividades.

\begin{tabular}{|c|c|c|c|c|c|c|c|c|c|}
\hline \multirow{2}{*}{ Rocas } & \multicolumn{7}{|c|}{ Cuadrículas } & \multirow{2}{*}{ Total } & \multirow{2}{*}{$\%$} \\
\hline & $\mathrm{A}_{2}$ & B2 & $\mathrm{B}_{3}$ & $\mathrm{C}_{2}$ & D2 & E2 & $F_{2}$ & & \\
\hline Caliza s. & o & o & o & 7 & 2 & o & o & 9 & 1,75 \\
\hline Cuarzo & o & o & o & 8 & 3 & o & 1 & 12 & 2,33 \\
\hline Basalto & o & o & 1 & o & o & o & o & 1 & 0,19 \\
\hline Dolomía s. & o & $\mathrm{o}$ & o & 1 & o & o & o & 1 & 0,19 \\
\hline Diabasa & o & o & o & o & 1 & o & o & 1 & 0,19 \\
\hline Pelita s. & o & o & o & 4 & 1 & o & o & 5 & 0,97 \\
\hline OFB & 2 & 9 & 30 & 104 & 40 & o & 6 & 191 & 37,01 \\
\hline OGSB & 9 & 14 & 40 & 162 & 63 & 1 & 2 & 291 & 56,5 \\
\hline Indet. & o & 0 & o & 3 & 1 & o & o & 4 & 0,77 \\
\hline Total & 11 & 23 & 71 & 289 & 111 & 1 & 9 & 515 & 100 \\
\hline$\%$ & 2,13 & 4,47 & 13,8 & 56,12 & 21,55 & 0,19 & 1,74 & 100 & \\
\hline
\end{tabular}

Tabla 2. Materias primas identificadas en todo el conjunto lítico del sitio. Referencias: Caliza s.: Caliza silicificada, Dolomía s.: dolomía silicificada, Pelita s.: pelita silicificada, OFB: Ortocuarcitas de la Formación Balcarce, OGSB: Ortocuarcitas del Grupo Sierras Bayas, Indet.: indeterminadas.

En estas rocas, también se recuperó un núcleo con lascados aislados. En esta pieza, las extracciones de los lascados no siguen un ordenamiento determinado durante el desbaste del núcleo y aún resta volumen de roca por explotar. Las otras variedades de rocas que se presentan en bajos porcentajes, poseen calidades diferentes para la talla y proceden de áreas geográficas diversas. Dentro de este grupo de rocas se destacan las calizas silicificadas por su gran distancia a las fuentes de aprovisionamiento (Bonomo y Blasi 2010; Flegenheimer, Bayón, Valente, Femeninas y Valente, 2003; Loponte, Tchilinguirian y Sacur Silvestre, 2011; Nami, 2017). Los artefactos de esta roca silícea son pequeños (lascas y microlascas) y productos derivados de las etapas de regularización y mantenimiento de instrumentos. En el conjunto también se reconocieron en baja frecuencia lascas y microlascas de diabasa, dolomía, cuarzo y pelita silicificada. Este patrón en el uso de los recursos líticos es un aspecto recurrente en la tecnología lítica de los sitios de la transición Pleistoceno-Holoceno de Tandilia oriental (Bonnat, 2016). 


\begin{tabular}{|c|c|c|c|c|c|c|c|}
\hline Rocas & Clase & $\mathrm{C}_{2}$ & D2 & E2 & F2 & Total & $\%$ \\
\hline \multirow{2}{*}{ Caliza s. } & Lasca & 3 & 1 & o & o & 4 & 0,99 \\
\hline & Microlasca & 4 & 1 & o & $\mathrm{o}$ & 5 & 1,23 \\
\hline \multirow{2}{*}{ Cuarzo } & Lasca & 7 & 2 & o & 1 & 10 & 2,47 \\
\hline & Microlasca & 1 & 1 & o & $\mathrm{o}$ & 2 & 0,49 \\
\hline Diabasa & Lasca & 0 & 1 & $\mathrm{o}$ & $\mathrm{o}$ & 1 & 0,25 \\
\hline Dolomia s. & Lasca & 1 & o & $\mathrm{o}$ & o & 1 & 0,25 \\
\hline \multirow{3}{*}{ OFB } & Lasca & 90 & 37 & o & 5 & 132 & 32,6 \\
\hline & Microlasca & 13 & o & o & $\mathrm{o}$ & 13 & 3,21 \\
\hline & Núcleo & 1 & 0 & 0 & 0 & 1 & 0,25 \\
\hline \multirow{3}{*}{ OGSB } & Lasca & 105 & 46 & 1 & 2 & 154 & 38,02 \\
\hline & Microlasca & 57 & 15 & o & $\mathrm{o}$ & 72 & 17,78 \\
\hline & Instrumentos & o & 1 & 0 & o & 1 & 0,25 \\
\hline \multirow{2}{*}{ Pelita s. } & Lasca & 1 & 1 & o & o & 2 & 0,49 \\
\hline & Microlasca & 3 & $\mathrm{o}$ & o & o & 3 & 0,74 \\
\hline \multirow{2}{*}{ Indet. } & Lasca & 2 & $\mathrm{o}$ & o & $\mathrm{o}$ & 2 & 0,49 \\
\hline & Microlasca & 1 & 1 & o & o & 2 & 0,49 \\
\hline \multicolumn{2}{|l|}{ Total } & 289 & 107 & 1 & 8 & 405 & 100 \\
\hline \multicolumn{2}{|l|}{$\%$} & 71,36 & 26,42 & 0,25 & 1,97 & 100 & \\
\hline
\end{tabular}

Tabla 3: Materias primas y clases artefactuales identificadas en la ocupación temprana del sitio Cueva Alí Mustafá. Referencias: Caliza s.: Caliza silicificada, Dolomía s.: dolomía silicificada, OFB: Ortocuarcitas de la Formación Balcarce, OGSB: Ortocuarcitas del Grupo Sierras Bayas, Pelita s.: pelita silicificada, Indet.: indeterminadas.

\section{Discusión y conclusiones}

A partir de los datos expuestos sobre el sitio Cueva Alí Mustafá, es posible plantear de modo preliminar algunas características singulares registradas en su contexto temprano y en su relación con el paisaje serrano. Una clave para comprender su posible función, surge del lugar ventajoso que ocupó esta cueva en el extremo del cañadón, desde donde sus ocupantes podían visualizar la casi totalidad del valle del arroyo San Pedro (Figura 2). Esta condición de mirador habría permitido controlar visualmente la circulación de otros grupos humanos y/o de los movimientos de los animales. Por otro lado, su baja visibilización habría limitado su identificación desde el exterior. Estas características, pudieron alentar su reutilización como paradero en los derroteros de grupos pequeños de cazadores-recolectores que atravesaban los valles o como un lugar complementario para quienes acamparon durante el mismo período en Cueva Tixi, distante una centena de metros.

Como se expresó, se reconocen dos principales ocupaciones, una temprana y otra datada en el Holoceno medio. El subconjunto tecnológico recuperado dentro de la cueva corresponde a la transición Pleistoceno-Holoceno temprano, caracterizado por la diversidad de rocas transportadas al sitio. Estas variedades se registraron en los desechos de la talla (lascas internas y numerosos microdesechos). Estas condiciones sugieren que la producción de artefactos líticos estuvo relacionada a las últimas etapas de la cadena operativa, es decir a la formatización final y/o al mantenimiento de 
instrumentos. Similares características se identificaron en ocupaciones efímeras del mismo período en otros sitios de Tandilia oriental como en: Alero El Mirador, Cueva La Brava, Amalia, Sitio 2, Localidad Lobería I, Abrigo la Grieta y Cueva Burucuyá (Bonnat, 2016). En tanto, el subconjunto recuperado en las cuadrículas del vestíbulo (A2-B2-B3) con cronología del Holoceno medio, sugiere diferencias en la gestión de los recursos líticos. La talla se produjo casi exclusivamente sobre ortocuarcitas (OGSB y OFB) (Tabla 2), cuyas evidencias son centralmente lascas. Este aspecto coincide con el patrón tecnológico analizado en otras ocupaciones del mismo período en sitios muy cercanos (Donadei, 2017; Mazzanti et al., 2013;). Si bien los dos subconjuntos pertenecen a ocupaciones distantes cronológicamente, el tipo de función dada a este reparo fue similar al utilizarse como paradero de corta duración. En el área centrooriental de Tandilia se registraron dos reparos rocosos pequeños con ocupaciones humanas efímeras que contenían materiales líticos. Se trata de los sitios Cueva Zoro y Los Helechos con dataciones en el rango ca.10200 a 9600 años AP y adquieren importancia local porque también se destaca la ubicación en el paisaje de sierras con dominio visual de la zona (Flegenheimer, Mazzia y Weitzel, 2015).

En la Cueva Alí Mustafá las actividades de talla ocurrieron en su interior pero en el sector más cercano al ingreso donde hay mayor incidencia de la luz natural. En tanto, inmediatamente hacia su interior donde la iluminación es muy escasa, se registró la gran área destinada a la producción de combustión mediante fogones del tipo cubeta que conservaron en sus rellenos abundante carbón. Algunos se hallaron en diferentes profundidades o con ligeras superposiciones (Figura 5B). Lo cual indicaría la necesidad o interés en reiterar las mismas prácticas en el área oscura de esta cavidad. Estos fogones estaban rodeados por un sedimento que presentaba numerosas inclusiones de trozos de carbones dispersos. Esta característica se vincula a las acciones de limpieza de los fogones (Caruso Fermé, 2013) o también pudo originarse por el desplazamiento causado por agentes erosivos (viento, agua, animales). El problema principal, de difícil resolución, es determinar las funciones de los nueve fogones concentrados en una misma área, considerando que, además, hay indicios observados en el perfil noreste que ampliarían este número. Tradicionalmente los fogones se produjeron en cuevas para generar calor, iluminar, cocinar alimentos o bien para actividades de producción de humaredas con fines de conservación de alimentos o de otros materiales orgánicos. La determinación de los recursos vegetales seleccionados como leña podría ayudar a determinar potenciales funciones. En esta primera etapa de las investigaciones, se considera que el área de combustión se relaciona con el interés de producir calor e iluminación de la cámara. Las condiciones frías del postglacial quedan en evidencia porque en todos los reparos con mayor integridad estratigráfica se registraron numerosas áreas de combustión. No obstante lo cual, la ubicación del área general de combustión en el sector del techo más bajo, plantea la posibilidad que pudieron generar situaciones de humareda interna. La producción de fogones en cubetas es una característica de los grupos de cazadores-recolectores que durante el postglacial ocuparon también otros reparos rocosos en este mismo macizo serrano, como lo indica Cueva Burucuyá y Abrigo Los Pinos, o en parajes cercanos con el ejemplo de Cueva La Brava. La buena conservación de varios fragmentos de la leña utilizada en esas estructuras de combustión, permitió conocer algunos de los recursos vegetales recolectados localmente. Se logró una promisoria base de datos sobre la diversidad de recursos vegetales presentes en ese período con clima fluctuante entre ciclos de aridez y humedad (Brea et al., 2014; Martínez y Mazzanti, 2017).

En síntesis, los reparos rocosos del área fueron ocupados respondiendo a una misma red de circulación propia de la dinámica social del período, registrada en las dos cuencas de ocupación de la sierra La Vigilancia (Mazzanti y Bonnat, 2013). Este modelo propuso la necesidad de vincular las ocupaciones en cuevas y abrigos, tanto recurrentes como efímeras, con las condiciones de esos emplazamientos y el paisaje serrano. En esa 
peculiar planificación de los lugares donde asentarse, se destacan en su localización geográfica porque desde ellos fue posible controlar y/o dominar visualmente ciertos recursos naturales inmediatos o cercanos. Todos estos aspectos fueron estratégicos en la vida cotidiana de estas sociedades de cazadores-recolectores tempranos. Este patrón de asentamiento se produjo en estas sierras orientales, adyacentes al océano Atlántico, únicamente durante el rango temporal de $c a .9000$ a 10700 años AP. Las condiciones de estas redes de asentamiento/movilidad fueron analizadas desde el concepto de cuencas de ocupación (Criado Boado, 1999) cuya puesta a prueba facilitó comprender la organización espacial de los sitios de la transición Pleistoceno-Holoceno. En este contexto microregional, la Cueva Alí Mustafá aporta un nuevo sitio a este modelo de organización del espacio.

\section{Agradecimientos}

A la ANPCyT por financiar esta labor mediante el PICT 2013 (1979), a la Facultad de Humanidades (UNMDP) por brindar y acondicionar el lugar de trabajo del Laboratorio de Arqueología. A Carlos Quintana por su labor en el campo y en la identificación de la fauna moderna. Va nuestro agradecimiento a Mario Russa por su constante apoyo y hospitalidad. A los estudiantes de la carrera de Historia (UNMDP): Camila Petruzzi, Jeremías Barroso y Ángel Bigarani por trabajar activamente en las excavaciones arqueológicas. A los propietarios de la estancia por autorizar la permanencia del grupo de investigadores. 


\section{Q Referencias citadas}

》 Aschero, C. (1975). Ensayo para una clasificación morfológica de artefactos líticos aplicada a estudios tipológicos comparativos. Informe CONICET. Manuscrito inédito.

"Aschero, C. (1983). Registro de códigos para atributos descriptivos aplicados a artefactos líticos. Informe CONICET. Manuscrito inédito.

» Bellelli, C., Guraieb, G. y García, J. (1985-1987). Propuesta para el análisis y procesamiento por computadora de desechos de talla lítica (DELCO - Desechos Líticos Computarizados). Arqueología Contemporánea, 2(1), 36-53.

" Bonnat, G. F. (2016). Análisis de la organización de la Tecnología Lítica de Grupos Cazadores-recolectores tempranos del área de Tandilia Oriental (Buenos Aires). (Tesis Doctoral inédita), Facultad de Ciencias Sociales, Universidad Nacional del Centro de la Provincia de Buenos Aires, Argentina.

» Bonnat, G. F., Mazzanti, D. L. y Martínez, G. A. (2015). Tecnología lítica y contexto geoarqueológico de la ocupación temprana del Sitio 2 de la localidad arqueológica Amalia, provincia de Buenos Aires (Argentina). Revista del Museo de Antropología, 8(2), 21-32.

"Bonomo, M. y Blasi, A. (2010). Base regional de recursos líticos del Delta del Paraná. Estudio petrográfico de artefactos y afloramientos en el sur de Entre Ríos. CazadoresRecolectores del Conos Sur. Revista de Arqueología, 4, 17-41.

» Brea, M., Mazzanti, D. y Martínez, G. (2014). Selección y uso de los recursos madereros en cazadores-recolectores de la transición Pleistoceno-Holoceno y Holoceno medio, sierras de Tandilia oriental, Argentina. Revista del Museo Argentino de Ciencias Naturales. Nueva serie, 16(2), 129-141.

"Caruso Fermé, L. (2013). Los recursos vegetales en arqueología. Estrategias de muestreo y estudio del material leñoso. Buenos Aires: Editorial Dunken.

»Chumbita, H. (2000). Jinetes rebeldes. Historia del bandolerismo social en la Argentina. Buenos Aires: Editorial Vergara.

»Colobig, M. M. (2011). Estudios paleobotánicos en sitios del borde oriental de Tandilia (Buenos Aires, Argentina). Pautas de aprovechamiento y uso de recursos vegetales en las sociedades de cazadores-recolectores. (Tesis Doctoral inédita), Universidad Nacional de Rosario, Argentina.

»Criado Boado, F. (1999). Del terreno al Espacio: Planteamientos y perspectivas para la Arqueología del Paisaje. Criterios y Convenciones en Arqueología del Paisaje, CAPA 6,1-58.

»Dágata, J. (1987). Sucedió en el Valle. Buenos Aires: Editorial Colihue.

»Donadei, J. P. (2017). Gestión de la materia prima y estrategias de talla durante el Holoceno medio, Tandilia oriental: el caso de Cueva Tixi (Argentina). Comechingonia, Revista de Arqueología, 21(2), 379-399.

" Flegenheimer, N., Bayón, C., Valente, M., Femeninas, J. y Valente, J. (2003). Long distance tool stone transport in the Argentine Pampas. Quaternary International, 109-110, 49-64.

» Flegenheimer, N., Mazzia, N. y Weitzel, C. 2015. Landscape and rocks in the east-central portion of the Tandilia range (Buenos Aires Province, Argentina), PaleoAmerica, 1(2), 163-180.

"Loponte, D., Tchilinguirian, P.ySacur Silvestre, R. (2011). Caracterización de afloramientos de calizas silicificadas de la provincia de Entre Ríos (Argentina) y su vinculación con los circuitos de abastecimiento prehispánico. En M. Feuillet, M. Terzaghi, B. Colasurdo, J. Sartori y S. Escudero (Eds.), Avances y Perspectivas en la Arqueología del Nordeste (125139). Santa Fe: Municipalidad de Santo Tomé. 
" Luna, L., Aranda, C. y Quintana, C. (2017). Middle and Late Holocene micromammal pathologies from Cueva Tixi (Tandilia range, Buenos Aires Province, Argentina). International Journal of Paleopathology, 18, 82-91.

" Martínez, G. A. (2007). Procesos de formación de sitios en reparos rocosos de Tandilia. Cazadores-recolectores del Cono Sur. Revista de Arqueología, 2, 105-127.

» Martínez, G. A. y Mazzanti, D. L. (2017). Evidencia geoarqueológica de la transición Pleistoceno-Holoceno en reparos rocosos de Tandilia oriental (Provincia de Buenos Aires). Relaciones de la Sociedad Argentina de Antropología, XLII, 83-106.

» Martínez, G. A., Mazzanti, D. L., Quintana, C., Zucol, A., Colobig, M., Hassan, G., Brea, M. y Passeggi, E. (2013). Geoarchaeological and Paleoenvironmental context of the human settlement in the Eastern Tandilia Range, Argentina. Quaternary International, 299, 23-37

» Mazzanti, D. L. (1993). Investigaciones arqueología en el sitio Cueva Tixi (Provincia de Buenos Aires, Argentina). Etnia, 38/39, 125-163.

» Mazzanti, D. L. (1995-96). Arqueología del Borde oriental de Tandilia: cambios y continuidades en el uso del espacio. Shincal. Revista de la Escuela de Arqueología, 5, 7-25.

» Mazzanti, D. L (2007). Arqueología de las Relaciones Interétnicas en las Sierras de Tandilia. (Tesis Doctoral inédita), Facultad de Filosofía y Letras, Universidad de Buenos Aires, Argentina.

» Mazzanti, D. L. y Bonnat, F. (2013). Paisajes arqueológicos y cazadores-recolectores de la transición Pleistoceno-Holoceno. Análisis de las cuencas de ocupación en Tandilia oriental, Provincia de Buenos Aires, Argentina. Relaciones de la Sociedad Argentina de Antropología, XVIII(2), 521-541.

» Mazzanti, D. L., Martínez, G. A., Colobig, M. M., Zucol, A. F., Passeggi, E., Brea, M., Bonnat G. F., Hassan, G., Soria, J. L., Vera, J. A. y Quintana, C. A. (2013). Avances en los estudios arqueológicos, geoarqueológicos y paleoambientales en las sierras de Tandilia. Resultados preliminares en Alero El Mirador y Abrigo La Grieta. Revista del Museo de La Plata, Sección Antropología, 13(87), 59-76.

» Mazzanti, D. L. y Porto López J. M. (2007). Caracterización Petrográfica y Estructural de Cerámicas Arqueológicas de las sierras de Tandilia. En B. Cremonte y N. Ratto (Comps.), Cerámicas Arqueológicas: Perspectivas arqueométricas para su análisis e interpretación (97122). Jujuy: Editorial de la Universidad Nacional de Jujuy.

»Mazzanti, D. L. y Quintana, C. (2001). Cueva Tixi: Cazadores y Recolectores de las Sierras de Tandilia. I. Geología, Paleontología y Zooarqueología. Publicación Especial 1. Mar del Plata: LARBO-UNMDP.

» Nami, H. (2017). Silcrete as a valuable resource for stone tool manufacture and its use by Paleo-American hunter-gatherers in southeastern South America. Journal of Archaeological Science: Reports, 15, 539-560.

»Quintana, C. A. (2016). Tafonomía de los microvertebrados del sitio arqueológico Cueva Tixi (Pleistoceno tardío-Holoceno tardío) Tandilia oriental, provincia de Buenos Aires, Argentina. Publicación Electrónica de la Asociación Paleontológica Argentina, 16(1), 14-51.

»Zucol, A. F., Brea, M., Osterrieth, M. y Martínez, G. (2002). Análisis fitolítico de un horizonte sedimentaria del Sitio 2 de la localidad Arqueológica Amalia. En: D. Mazzanti, M. Berón y F. Oliva (Eds.), Del Mar a los Salitrales. Diez Mil Años de Historia Pampeana en el Umbral del Tercer Milenio (355-365). Mar del Plata: Universidad Nacional de Mar del Plata. 